\title{
INTERPOSIÇÃO DE ENXERTO ILÍACO DE DOADOR PARA REVASCULARIZAÇÃO DE IMPLANTES EM TRANSPLANTE ORTOTÓPICO DE FÍGADO: DESCRIÇÃO DA CASUÍSTICA EM 745 TRANSPLANTES REALIZADOS E REVISÃO DA LITERATURA
}

\author{
Interposition of donor iliac graft to revascularization of liver in orthotopic transplantation: \\ description of 745 transplants sampling performed and literature review
}

\author{
Rafael Leite Nunes, Elaine Cristina de Ataide, Milena Silva Garcia, Simone Reges Perales, Raquel Silveira Stucch, \\ Andre Berton Verdan, llka de Fátima Santana Ferreira Boin
}

\begin{abstract}
RESUMO
Trombose da artéria hepática é complicação importante em transplantes hepáticos, permanecendo como grave causa de falha do enxerto. O tratamento envolve revascularização, feita com técnicas endovasculares ou reconstrução arterial. Em casos como presença de trombose, a reconstrução alternativa da artéria hepática deve ser considerada, e nesse sentido o enxerto da artéria ilíaca do doador tem sido utilizado como elemento de reconstrução arterial. Objetivou-se revisar a literatura e descrever a casuística onde foram utilizados enxertos arteriais com transposição de artéria ilíaca para revascularização dos implantes em transplante ortotópico de fígado, da Unidade de Transplante Hepático da Universidade Estadual de Campinas, no período de 1998 a 2015. Foram realizados 15 transplantes com necessidade de enxerto arterial, sendo oito casos do sexo masculino, com idade média de 50 anos. As indicações para o uso do enxerto foram: sete retransplantes por trombose da artéria hepática precoce (47\%), cinco transplantes com artéria hepática trombosada já no primeiro implante (35\%), um retransplante por trombose arterial tardia (6\%), um retransplante por complicações biliares (6\%), um paciente por lesão arterial pós-quimioembolização ainda no pré-operatório com inviabilidade da utilização de tronco celíaco (6\%). O tempo cirúrgico médio foi de 420 minutos, média de transfusão de concentrados de hemácias foi de cinco unidades. A sobrevida pós-procedimento em seis meses foi $60 \%$ e em um ano $53 \%$. A literatura corrobora o bom prognóstico observado em nossos casos, apontando essa manobra como segura e eficiente, com alguns estudos apoiando-a como técnica de escolha em casos de retransplante por trombose de artéria hepática.
\end{abstract}

Keywords: Transplante Hepático; Enxerto de Artéria llíaca; Trombose da Artéria Hepática.

Instituição

Faculdade de Ciências Médicas - Universidade Estadual de Campinas UNICAMP, Campinas/SP

\section{Correspondência}

Elaine Cristina Ataide

Centro de Diagnóstico de Doenças do Aparelho Digestivo - Gastrocentro/ UNICAMP - Unidade de Transplante Hepático

Rua Carlos Chagas, n420 - Cidade Universitária CEP 13.083-878 Campinas/SP

Tel.: (19) 99699-6806

E-mail: elainebataide@gmail.com

Recebido em: 26/10/2015

Aceito em: 10/12/2015

\section{INTRODUÇÃO}

A trombose da artéria hepática (TAH) é complicação importante em transplantes hepáticos. Grodzicki et al. (2011) descrevem uma taxa de mortalidade de $58 \%$, na presença dessa complicação. ${ }^{1}$ Vários são os fatores conhecidos para o desenvolvimento da THA, dentre eles, a literatura aponta como fatores de risco relacionados à técnica, as variações anatômicas, como a presença de vasos artérias pequenos e a necessidade de reconstrução arterial complexa pela presença de vasos aberrantes. ${ }^{2,3}$ 
Rafael Leite Nunes, Elaine Cristina de Ataide, Milena Silva Garcia, Simone Reges Perales, Raquel Silveira Stucch, Andre Berton Verdan,

Em relação aos fatores não relacionados à técnica, os estudos apontam como causas de trombose a presença de arteriosclerose na artéria hepática, fatores imunológicos como a presença de anticorpos anticardiolipina (ACA), o uso de implantes de doadores com status sorológico positivo para Citomegalovírus (CMV) em paciente com sorologia negativa, e o tabagismo persistente..$^{2-4}$

O tratamento da THA envolve revascularização que pode se dar com uso de técnicas endovasculares ou com uso de reconstrução arterial. As técnicas endovasculares incluem trombólise, sistêmica ou diretamente aplicada na artéria hepática, e o uso de stents. Esses procedimentos ainda em estudo são melhor empregados em pacientes assintomáticos e ainda tem uso limitado pelos riscos envolvidos, como a hemorragia. ${ }^{5}$

Em casos como presença de trombose, arteriosclerose entre outros elementos, o uso da reconstrução alternativa da artéria hepática deve ser considerado. ${ }^{6}$ Nesse sentido, a artéria ilíaca retirada do doador tem sido utilizada como elemento de reconstrução arterial. ${ }^{6-8}$

No que tange ao uso de próteses vasculares para a reconstrução arterial, existem poucos estudos comparando seu uso ao de enxertos arteriais. Entretanto, existem relatos de casos do uso de próteses, com boa evolução dos pacientes. ${ }^{9,10}$

Neste trabalho, objetivou-se revisar a literatura e descrever a casuística onde foram utilizados enxertos arteriais com transposição da artéria ilíaca para revascularização dos implantes em transplante ortotópico de fígado (TOF), na Unidade de Transplante Hepático da Universidade Estadual de Campinas, no período de 1998 a 2015.

\section{MÉTODOS}

Neste trabalho, analisaram-se os casos de TOF no período de 1998 a 2015. Nesse período, foram realizados 745 transplantes. Selecionaram-se os casos em que se utilizou a interposição do enxerto ilíaco para revascularização do fígado implantado, totalizando 15 casos. Foram selecionados dados quanto ao sexo e à idade dos pacientes envolvidos, tempo médio de procedimento cirúrgico, número de concentrados transfundidos de hemácias, sobrevida em seis meses e um ano. Os dados obtidos foram submetidos à estatística descritiva.

A técnica utilizada foi a interposição do enxerto de artéria ilíaca do doador entre a aorta (entre as artérias renais e a artéria mesentérica superior) e o tronco celíaco do doador (Figuras 1, 2 e 3). É importante destacar que em três casos, foram usados outros condutos arteriais: em um caso foi utilizada a artéria gastroduodenal do doador, em um caso a artéria escolhida foi a esplênica do doador e em um terceiro caso, utilizou-se prótese de politetrafluoroetileno (PTFE).

Figura 1 - Aspecto intraoperatório do enxerto de artéria ilíaca

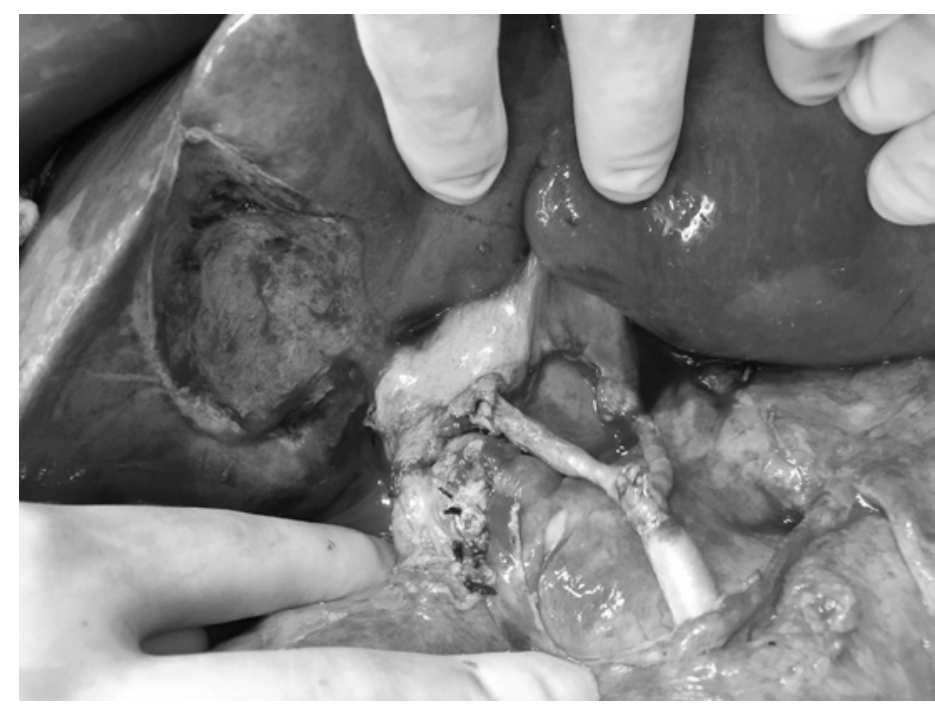

Figura 2 - Anastomose do enxerto de artéria ilíaca do doador na aorta do receptor

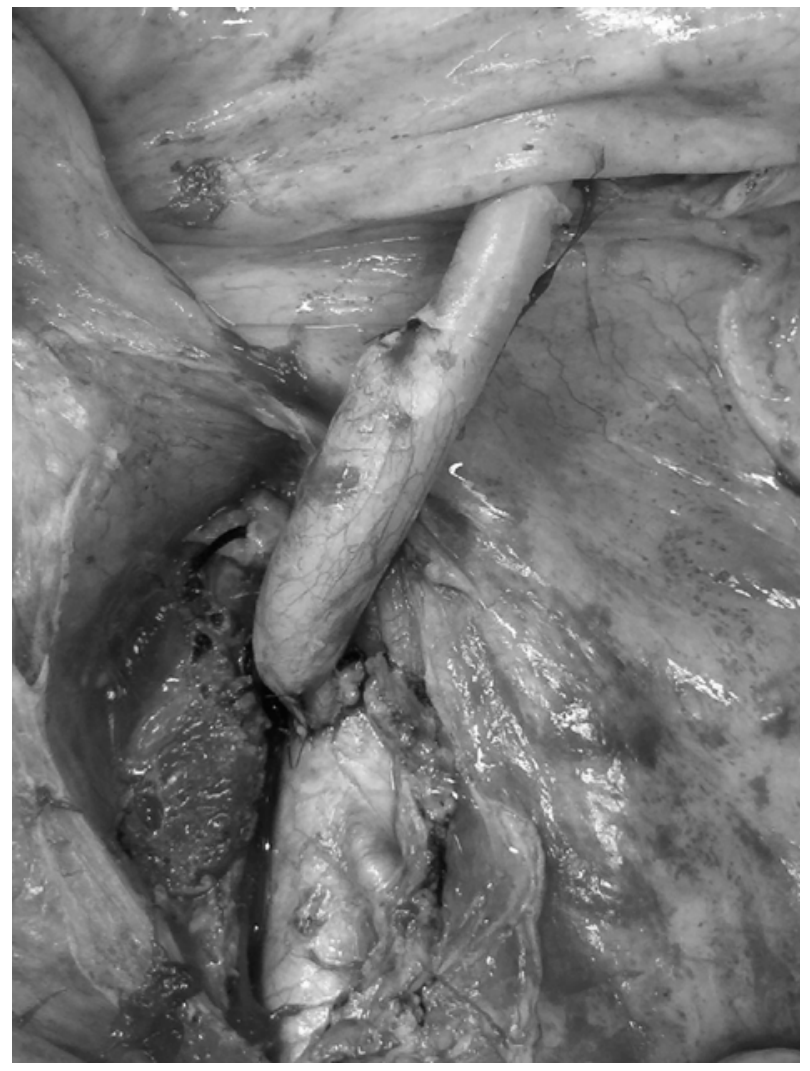


Interposição de enxerto ilíaco de doador para revascularização de implantes em transplante ortotópico de fígado: descrição da casuística em 745 transplantes realizados e revisão da literatura

Figura 3 - Imagem tomográfica demonstrando a perviedade do enxerto de artéria ilíaca

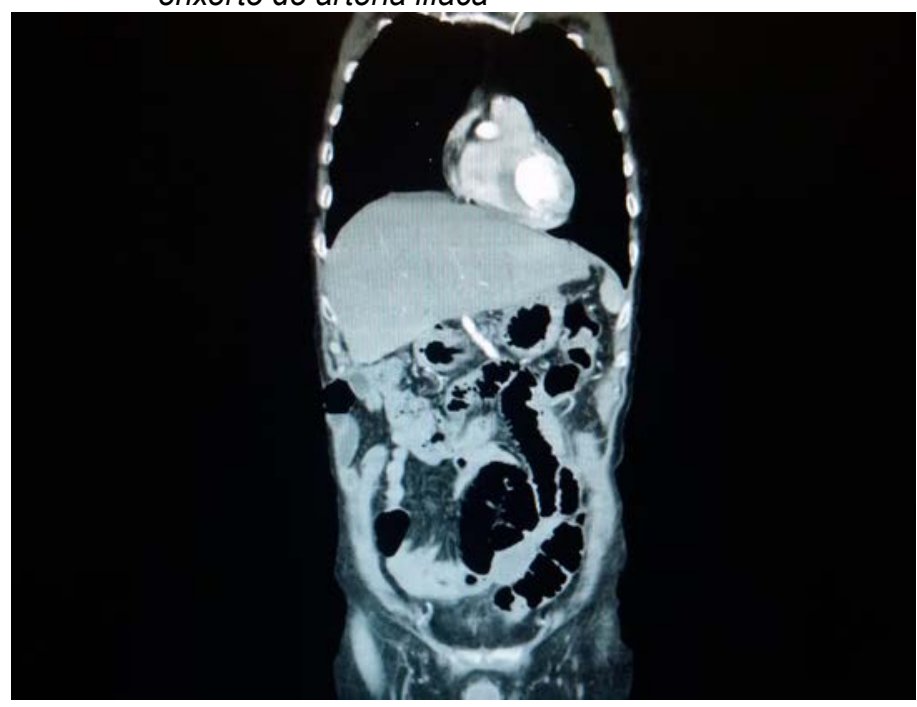

\section{RESULTADOS}

No período analisado, foram realizados 15 transplantes de fígado com necessidade de enxerto arterial. Tratavam-se de oito $(53 \%)$ casos do sexo masculino e sete $(47 \%)$ do sexo feminino. A idade média foi de 50,8 anos, com idade mínima de 39 e a máxima de 65 .

As indicações para 0 uso do enxerto foram: sete retransplantes por trombose da artéria hepática após o primeiro implante $(47 \%)$, cinco transplantes com artéria hepática trombosada já no primeiro implante (35\%), um retransplante por trombose arterial tardia (6\%), um retransplante 18 meses após o primeiro transplante por complicações biliares (6\%), um paciente por lesão arterial pós-quimioembolização ainda no pré-operatório com inviabilidade da utilização de tronco celíaco (6\%) (Tabela 1).

Tabela 1 - Indicações do uso de enxertos arteriais

\begin{tabular}{llc}
\hline \multicolumn{2}{c}{ Indicação } & Porcentagem \\
\hline Retransplante por TAH Preoce & $47 \%(7 / 15)$ \\
TAH no primeiro transplante & $35 \%(5 / 15)$ \\
& Lesão Arterial Pós- & $6 \%(1 / 15)$ \\
\multirow{2}{*}{ Outras causas } & Quimioembolização & $6 \%(1 / 15)$ \\
& Complicações Biliares & $6 \%(1 / 15)$ \\
\hline
\end{tabular}

O tempo cirúrgico médio foi de 420 minutos e a média de transfusão de concentrados de hemácias foi de cinco unidades. A sobrevida pós-procedimento em seis meses foi $66 \%$, em um ano $53 \%$. É importante destacar que, dos pacientes incluídos nessa casuística, quatro (26\%), ainda não completaram um ano de realização, o que interferiu na análise da sobrevida em um ano (Tabela 2).
Tabela 2 - Índice de sobrevida após transplante utilizando enxerto de artéria ilíaca

\begin{tabular}{cc}
\hline Tempo de Sobrevida & Resultados \\
\hline 6 meses & $66 \%$ \\
Um ano & $53 \%$ \\
\hline
\end{tabular}

Em relação ao índice de complicações, dois (13\%) pacientes apresentaram novo episódio de trombose da artéria hepática, necessitando de novo retransplante. No que tange aos óbitos, cinco (33\%) pacientes faleceram em menos de 30 dias. Entretanto, nenhum desses pacientes apresentou complicações vasculares, sendo as causas de óbito coagulopatia, sepse e colangite.

\section{DISCUSSÃO}

Complicações vasculares como a trombose da artéria hepática e da veia cava podem culminar na perda do enxerto e óbito. ${ }^{11}$ Bekker et. al., em revisão da literatura de casuísticas de diferentes serviços, realizada através de bases de dado indexadas (MEDLINE, EMBASE, Science Citation Index), encontrou incidência média de $3,9 \%$ de trombose precoce da artéria hepática com taxa de mortalidade de $33,3 \%$, sendo maior em adultos. ${ }^{12}$

Ainda em relação à THA, numa revisão de 4234 transplantes realizados, um grupo da Califórnia relatou incidência de 5\% (203 casos) de trombose da artéria hepática. No total de casos com essa complicação, $17 \%$ necessitaram de retransplante como terapêutica inicial e $65 \%$ passaram por revisão de anastomose e trombectomia. Entretanto, desses últimos, em $78 \%$ a terapêutica inicial não foi efetiva e também foram submetidos a retransplantes. A presença de $\mathrm{TAH}$ reduziu a sobrevida do enxerto de aproximadamente $75 \%$ em um ano nos pacientes sem essa complicação para cerca de $25 \%$ nos com trombose $(p<0,001) .{ }^{13}$

Em nossa casuística, a principal indicação do uso de enxerto de artéria ilíaca do doador foi o retransplante por TAH (47\% dos casos), dado semelhante ao de outras casuísticas descritas na literatura, como Liu et. al., que também relatam THA como principal causa do uso de enxerto (22\% dos casos). ${ }^{14} \mathrm{Na}$ casuística de Muralidharan et. al., a porcentagem foi de $50 \% .{ }^{15}$

Ainda, nossos pacientes apresentaram sobrevida, em seis meses, de $66 \%$ e, em um ano, de $53 \%$. O índice de complicações vasculares foi de $13 \%$, representados por dois casos de novo episódio de TAH. As outras complicações encontradas (Sepse (6\%), Coagulopatia (20\%) e Síndrome Coronariana Aguda (6\%), complicações graves que levaram ao óbito, mas não se relacionaram ao uso de enxertos arteriais. 
Rafael Leite Nunes, Elaine Cristina de Ataide, Milena Silva Garcia, Simone Reges Perales, Raquel Silveira Stucch, Andre Berton Verdan, Ilka de Fátima Santana Ferreira Boin

Meyer et. al. revisaram 434 transplantes de sua casuística. Nesse grupo, 43 necessitaram de enxertos da artéria ilíaca para revascularização. Não encontraram diferenças em complicações vasculares na comparação entre o grupo de pacientes submetidos a anastomose arterial standard (anastomose entre a artéria hepática comum do doador com o tronco celíaco do receptor) e o grupo que recebeu enxerto de artéria ilíaca. ${ }^{16}$

Encontraram incidência maior de trombose da artéria como complicação em pacientes que foram retransplantados e que foi utilizado, no retransplante, enxerto de artéria ilíaca $(35,7 \%$ de trombose nos pacientes com uso de enxerto e $4,7 \%$ nos que foram submetidos a anastomose standard no retransplante $(p=0,02)) \cdot{ }^{16} \mathrm{O}$ índice de THA nesse estudo foi maior do que o encontrado no presente estudo.

Em estudo realizado por $\mathrm{Ma}$ et. al. foram analisados 48 casos de pacientes submetidos a transplante utilizando enxertos de artéria ilíaca para reconstrução arterial em TOF. Nesse estudo, observou-se patência em 100\% das reconstruções. A sobrevida média foi de 3.9 anos. Não foram observadas complicações vasculares em nenhum dos casos. ${ }^{17}$
Revisando 582 TOF realizados entre 1986 e 2003, Liu et. al. relataram o uso de condutos arteriais (enxertos arteriais ou próteses vasculares) em 5,3\% dos casos, sendo que em $90 \%$ foi utilizada a artéria ilíaca do doador. A TAH foi a principal causa de necessidade de uso de condutos (sete casos dos 31 que necessitam de algum conduto arterial). Encontraram 10\% (três casos) de complicações relacionadas ao enxerto (dois casos de TAH e um caso de infecção relacionada à prótese Gore-Tex utilizada como conduto). Relatam ainda maior necessidade de retransplante no grupo que utilizou conduto arterial (42\% no grupo do enxerto (13/31) e 5\% no grupo sem condutos $(28 / 551)(p=0,001) .14$

\section{CONCLUSÃO}

Nesse sentido, a literatura corrobora os bons resultados encontrados em nossa casuística, que apresentou boa perfusão do implante e boa evolução pós-procedimento, com baixo índice de complicações relacionadas ao uso de enxertos arteriais. Esses resultados sugerem que 0 uso dessa manobra é seguro e eficiente, com alguns estudos apoiando-a como técnica de escolha em casos de retransplante por trombose de artéria hepática.

\section{ABSTRACT}

Hepatic artery thrombosis is an important complication in liver transplants, remaining a serious cause for graft failure. Treatment involves revascularization performed by endovascular techniques or arterial reconstruction. In cases such as presence of thrombosis, the alternative reconstruction of the hepatic artery must be considered, and in such sense, the graft from the iliac artery of donor has been used as arterial reconstruction element. The purpose was to review the literature and to describe series where it was used iliac artery arterial grafts for revascularization of the implants in orthotopic liver transplantation in the Liver Transplant Unit at the State University of Campinas from 1998 to 2015. It has been performed 15 transplants needing arterial graft: 8 cases of male, mean age 50 years. Indications to use the graft were: 7 retransplant thrombosis in early hepatic artery (47\%), 5 transplantations with hepatic artery already thrombosed in the first implant (35\%), 1 retransplantation for late arterial thrombosis $(6 \%), 1$ retransplantation due to biliary complications $(6 \%), 1$ patient due to post-chemoembolization arterial injury still preoperatively with impossibility of using the celiac trunk (6\%). The mean operative time was 420 minutes, mean transfusion of packed red blood cells was 5 units. Post-procedure survival at 6 months was $60 \%$ at 1 year and $53 \%$. The literature confirms the good prognosis observed in our cases pointing this maneuver as safe and effective, with some studies supporting it as technique of choice in cases of retransplantation due to hepatic artery thrombosis.

Keywords: Liver Transplantation; Iliac Artery Graft; Hepatic Artery Thrombosis. 


\section{REFERÊNCIAS}

1. Grodzicki M, Anysz-grodzicka A, Remiszewski P, Cieslak B, Kotulski M, Kalinowski $P$, et. al. Treatment of early hepatic artery thrombosis after liver transplantation. Transplant Proc. 2011;43:3039-42.

2. Nemes B, Gaman G, GelleY F, Doros A, Zadori G, Gorog D, et. al. Technical risk factors for hepatic artery thrombosis after orthotopic liver transplantation: the hungarian experience. Transplant Proc. 2013;45:3691-4.

3. Mueller AR, Platz KP, Kremer B. Early postoperative complications following liver transplantation. Best Pract Res Clin Gastroenterol. 2004;18(5):881-900.

4. Pastacaldi S, Teixeira R, Montalto P, Rolles K, Burroughs AK Hepatic artery thrombosis after orthotopic liver transplantation: a review of nonsurgical causes. Liver Transpl. 2001;7(2):75-81.

5. Singhal A, Stokes K, Sebastian A, Wright HI, Kohli V. Endovascula treatment of hepatic artery thrombosis following liver transplantation. Transpl Int. 2010;23:145-256.

6. Del Gaudio M, Grazi GL, Ercolani G, Ravaioli M, Varotti G, Cescon $\mathrm{M}$, et. al. Outcome of hepatic artery reconstruction in liver transplantation with an iliac arterial interposition graft. Clin Transplant. 2005;19:399-405.

7. Mabrut JY, Abdullah SS, Rode A, Bourgeot JP, Eljaafari A, Baulieux J, et. al. Cryopreserved iliac artery allograft for primary arterial revascularization in adult liver transplantation. Clin Transplant. 2012;26:E12-E16.

8. Shimizu S, Onoe T, Ide K, Oshita A, Amano H, Kobayashi T., et. al. Complex vascular reconstruction using donor's vessel grafts in orthotopic liver transplantation. Transplant Proc. 2012,44:574-8.

9. Golse N, Spina A, Abdelaal A, Mennesson N, Feugier $P$, Dumortier J, et. al. Extra-anatomical hepatic artery reconstruction following post-embolization iatrogenic dissection and arterial anastomotic rupture in two liver transplant recipientes. Gastroenterol Clin Biol. 2010;34:111-4.
10. Mosna LC, Moon J, Hernandez F, Hodgkinson $P$, Fan J, Selvaggi G, et. al. Use of subcutaneous route and polytetrafluoroethylene grafts for arterial revascularization in liver transplantation. Liver Transpl. 2012;18(4):499-500.

11. Unal B, Gonultas F, Aydin C, Otan E, Kayaalp C, Yilmaz S. Hepatic artery thrombosis-related risk factors after living donor liver transplantation: single-center experience from turkey. Transplant Proc. 2013;45:974-7.

12. Bekker J, Ploem S, Jong KP. Early hepatic artery thrombosis after liver transplantation: a systematic review of the incidence outcome and risk factors. Am J Transplant. 2009;9:746-57.

13. Duffy JP, Hong JC, Farmer DG, Ghobrial RM, Yersiz $\mathrm{H}$, Hiatt JR, et. al. Vascular complications of orthotopic liver transplantation: experience in more than 4200 patients. J Am Coll Surg. 2009;208(5):896-903.

14. Liu T, Dilworth P, Sosef M, Wang C, Crawford M, Gallagher J, et. al. Arterial vascular conduits in adult orthotopic liver transplant recipientes. ANZ J Surg. 2006;76:64-67.

15. Muralidharan V, Imber C, Leelaudomlipi S, Gunson BK, Buckels JAC, Mirza DF, et. al. Arterial conduits for hepatic artery revascularization in adult liver transplantation. Transpl Int. 2004;17:163-8.

16. Meyer C, Riehm S., Perrot F, Cag M., Nizand M, Audet $\mathrm{M}$, et. al. Donor iliac artery used for arterial reconstruction in liver transplantation. Transplant Proc. 2000;32:2791.

17. Ma Y, Li Q, Ye Z, Zhu X, He X. Use of arterial conduit for arterial revascularization during liver and multivisceral transplantation. Chin Med J. 2011;124(19):2986-9. 